\title{
O EFEITO DA MUSCULAÇÃO NA TERCEIRA IDADE
}

\section{ARTIGO DE REVISÃO}

RABELO, Olga Alves ${ }^{1}$

SOUZA, Lucas Alves de ${ }^{2}$

CAETANO, Thaynara Alves ${ }^{3}$

SILVA, Sebastião Lobo da 4

SANTOS, Givanildo de Oliveira ${ }^{5}$

RABELO, Olga Alves. Et al. 0 efeito da musculação na terceira idade. Revista Científica Multidisciplinar Núcleo do Conhecimento. Ano 05, Ed. 09, Vol. 03, pp. 144152. Setembro de 2020. ISSN: 2448-0959, Link de acesso: https://www.nucleodoconhecimento.com.br/educacao-fisica/efeito-damusculacao

\section{RESUMO}

O envelhecimento é entendido como um conjunto de alterações biológicas que ocorrem com o decorrer do tempo, acarretando mudanças estruturais no corpo. Historicamente, envelhecer carrega um estereótipo social negativo baseado na errônea ideia de que o envelhecimento gera uma incompetência comportamental. Este trabalho teve como objetivo realizar uma revisão bibliográfica, através do

\footnotetext{
${ }^{1}$ Bacharel em Educação Física, pela Faculdade de Mauá Brasília - DF.

2 Bacharel em Educação Física, pela Faculdade de Piracanjuba - GO.

${ }^{3}$ Bacharel em Educação Física, pela Faculdade de Piracanjuba - GO.

${ }^{4}$ Doutorando em Educação Física, pela Universidade Católica de Brasília - DF.

${ }^{5}$ Mestre em Tecnologia de Alimentos, pelo Instituto Federal Goiano - Campus Rio Verde - GO.
} 
levantamento de artigos publicados em revistas indexadas nos últimos 10 anos (20092019), sobre os efeitos da prática de musculação na vida de idosos de ambos os sexos. De acordo com os resultados, os efeitos incluem benefícios cardiovasculares, desenvolvimento da capacidade aeróbia, melhora na composição corporal, aumento do equilíbrio, aumento da força e capacidade funcional, melhora nos índices de aptidão física e melhora dos componentes psicomotores. E de maneira geral, a musculação proporciona aumento na força muscular e na resistência do idoso, melhorando seu equilíbrio e a sua capacidade cognitiva.

Palavras-chave: Idoso, envelhecimento, musculação, exercícios físicos, sarcopenia.

\section{INTRODUÇÃO}

De acordo com Souza et al. (2014) houve a evolução populacional de forma contínua, passando por altas taxas de mortalidade e natalidade, porém, a expectativa de vida tem aumentado, havendo o aumento da população idosa. Conforme a quantidade de indivíduos que chega à terceira idade aumenta, crescem também os estudos que visam entender o processo de envelhecimento do ser humano, uma vez que o aumento da expectativa de vida demanda ações preventivas, restauradoras e reabilitadoras (ALMEIDA; PAVAN, 2010).

Tais estudos visam atribuir qualidade de vida a essa população, que não só tem possibilidades de viver mais, porém merece e pode viver com qualidade, dignidade e, principalmente, estimulada para a superação de pequenos desafios. Historicamente, o processo de envelhecer carrega um estereótipo social negativo baseado na errônea ideia de que o envelhecimento gera uma incompetência comportamental. Por isso é fundamental que a prática de exercícios físicos seja incentivada tanto no âmbito social quanto familiar (CORAZZA, 2001).

Este trabalho teve como objetivo realizar uma revisão bibliográfica, através do levantamento de artigos publicados em revistas indexadas nos últimos 10 anos, sobre os efeitos da prática de musculação na vida de idosos de ambos os sexos. 
A musculação pode ser uma grande aliada na promoção da qualidade de vida na terceira idade, pois já é comprovado que esta atividade promove um aumento notável na força muscular e resistência do idoso, melhora o equilíbrio, aumenta a capacidade mitocondrial e diminui a frequência cardíaca de repouso. Estes fatores são primordiais para que o indivíduo consiga realizar tarefas do seu dia a dia de maneira mais autônoma e independente, recuperando assim sua autoconfiança e autoestima.

\section{DESENVOLVIMENTO}

\section{O ENVELHECIMENTO}

O envelhecimento é entendido como um conjunto de alterações biológicas que ocorrem com o decorrer do tempo, acarretando mudanças estruturais no corpo. Este, se torna mais perceptível ao final da fase reprodutiva e envolve variáveis como a genética, o estilo de vida e doenças crônicas que interagem entre si influenciando a maneira pela qual o indivíduo envelhece e determinando também a idade que este indivíduo alcançará (ALMEIDA; PAVAN, 2010).

Segundo a Organização Mundial da Saúde, o envelhecimento pode ser classificado em quatro estágios cronológicos: meia idade de 45 a 59 anos; idoso de 60 a 74 anos, velho de 75 a 90 anos e muito velho de 90 anos em diante (WHO, 2019). Porém, a idade cronológica se mostra muitas vezes arbitrária uma vez que o envelhecimento é vivenciado de forma heterogênea pela população. Em função disso, outros indicadores também precisam ser levados em consideração como, por exemplo, a idade biológica, a idade psicológica e a idade social (AGUIAR et al., 2014).

Conforme Corazza (2001), a idade biológica diz respeito ao modo de vida que a pessoa leva, seus hábitos alimentares, a prática de exercícios físicos e o seu estado de saúde. Se o indivíduo possui hábitos de vida saudáveis, sua idade biológica será menor que a cronológica. Entretanto, se este possui fatores de risco para doenças cardiovasculares (diabetes, fumo, hipertensão, obesidade, sedentarismo), por consequência, sua idade biológica será maior que a cronológica, a idade psicológica está relacionada às capacidades individuais, mentais ou cognitivas, como a 
autoestima e autossuficiência, aprendizagem, memória e percepção. Já a idade social se refere à noção de sociedade muitas vezes refletida no que é e no que não é um comportamento apropriado para o indivíduo daquela faixa etária (CORAZZA, 2001).

Historicamente, o processo de envelhecer carrega um estereótipo social negativo baseado na errônea ideia de que o envelhecimento gera uma incompetência comportamental, devido ao sentimento de incapacidade alimentado pela sociedade, o idoso acaba se isolando e deixa de continuar desempenhando uma vida ativa, mesmo apresentando uma boa saúde (ALMEIDA; PAVAN, 2010).

O primeiro impacto do envelhecimento ocorre com a perda do seu papel na sociedade, crescendo dessa forma também a angústia, a decepção e o sofrimento deste indivíduo pelo fato deste não conseguir executar tarefas cotidianas com o mesmo desempenho de antes (ALMEIDA; PAVAN, 2010).

Em função disso, nos últimos anos vêm crescendo o interesse científico pela temática do envelhecimento, uma vez que há uma tendência ao envelhecimento global. Tais estudos visam atribuir qualidade de vida a essa população, que não só tem possibilidades de viver mais, porém merece e pode viver com qualidade, dignidade e, principalmente, estimulada para a superação de pequenos desafios.

Durante o processo de envelhecimento, em decorrência da redução da eficácia de um conjunto de processos fisiológicos, ocorre também um decréscimo do sistema neuromuscular e consequentemente a sarcopenia, ocorrendo a redução da flexibilidade, força, resistência e do equilíbrio estático e dinâmico. Essas alterações também afetam a marcha, o sistema visual, cardiorrespiratório, viscerais, neurológicos e imunológicos acabam acentuando as limitações na interação do idoso com o meio em que ele vive (KIRKWOOD et al., 2007).

O envelhecimento também é responsável pela redução na capacidade de promover torque articular com rapidez, o que é essencial na realização de atividades que exigem força moderada. $O$ torque é entendido como uma força que tende a rotacional ou girar um corpo, este é fundamental para nosso aparelho locomotor para que atividades 
como levantar-se de uma cadeira, manter o equilíbrio ao evitar obstáculos e subir escadas sejam executadas com autonomia (CANNON et al., 2007).

\section{O EXERCÍCIO FÍSICO NA TERCEIRA IDADE}

Sabe-se que a prática regular de exercícios físicos por indivíduos de qualquer idade é essencial, porém é na terceira idade que essa regularidade é melhor refletida. Dentre os diversos benefícios pode-se destacar a diminuição no risco de quedas e fraturas, a prevenção de doenças (hipertensão arterial, osteoporose, artrite, depressão), a diminuição da taxa de gordura corporal e perfil lipídico, a melhoria das capacidades físicas como força, equilíbrio e coordenação motora (DIAS et al., 2016).

Segundo Araújo (2017), a prática regular de exercícios físicos é capaz de promover melhoras em três esferas da vida do idoso. A primeira é a fisiológica, através do controle da glicose, melhora a qualidade do sono e melhora a capacidade física relacionada à saúde. A segunda é a psicológica, promovendo o relaxamento, a redução dos níveis de ansiedade e estresse, melhoras cognitivas e no estado de espírito. A terceira é a social, pois deixa os indivíduos mais seguros, melhorando sua integração tanto social quanto cultural.

A recomendação de exercícios físicos para idosos, por estudiosos do envelhecimento, baseava-se em exercícios aeróbios principalmente a caminhada, por ser um exercício de fácil execução e acessível a todas as classes sociais (AGUIAR et al., 2014). Em seguida, a inclusão de outras atividades aeróbias como a natação e a hidroginástica, porém estas menos acessíveis à população desfavorecida economicamente. Por outro lado, exercícios de força ou resistidos, como a musculação, não costumavam ser recomendados por profissionais da saúde, principalmente devido aos riscos que poderiam oferecer a idosos com hipertensão ou cardiopatia, patologias comuns em indivíduos acima dos 60 anos (AGUIAR et al., 2014).

No entanto, estudos como os de Almeida e Pavan (2010); Aguiar et al. (2014) e Dias et al. (2016) demonstraram que exercícios de musculação são seguros e eficazes para a população desta faixa etária. 


\section{OS BENEFÍCIOS DA MUSCULAÇÃO NA TERCEIRA IDADE}

O treinamento de força, através da musculação, se mostra uma das formas de intervenção que melhor resulta na manutenção e aumento da massa muscular, auxiliando na melhoria da aptidão física funcional do indivíduo idoso (DIAS et al., 2016).

Souza et al. (2014), fez um estudo comparativo com homens e mulheres (acima de 60 anos) praticantes de musculação e um grupo controle de idosos sedentários. O critério de inclusão utilizado para os participantes foi de estar praticando a atividade há mais de 12 meses com uma frequência mínima de 2 vezes por semana e o de exclusão pessoas com patologias cardiorrespiratória, metabólica ou neuromuscular, que dificultasse a execução dos protocolos de avaliação. Foram avaliados o índice de massa corporal, a força máxima de membros inferiores por meio do teste de uma repetição máxima (1RM), o desempenho de equilíbrio funcional através da escala de equilíbrio de Berg (EEB) composta por 14 itens comuns à vida diária e o teste Timed Up and Go (TUG) que avalia o equilíbrio dinâmico.

Observou-se que os idosos praticantes de musculação foram os que apresentaram resultados significativos quanto a força máxima nos membros inferiores e no TUG. Além disso, foi observada uma correlação moderada e significante entre o equilíbrio dinâmico com a força máxima de membros inferiores, nos estudos realizados por Souza et al. (2014). Por outro lado, outros trabalhos comparando idosos praticantes e não praticantes de musculação observaram que os indivíduos não praticantes apresentam EEB significativamente menor em relação aos praticantes (PEDRO e AMORIM, 2008; JAREK et al., 2010).

Outro estudo realizado apenas com mulheres idosas sedentárias, divididas em dois grupos, grupo controle e o grupo de treinamento, onde as idosas foram submetidas a treinamento resistindo definido por exercícios de membros superiores e inferiores, três vezes por semana, mostrou que após 12 semanas de intervenção houve um aumento de $58 \%$ na força média da parte superior do corpo, $68 \%$ de aumento na força média 
da parte inferior do corpo e aumento de $19 \%$ na capacidade cognitiva dessas mulheres (SMOLAREK et al., 2016).

Um estudo recente, publicado em 2018, objetivou avaliar a autonomia funcional de idosos praticantes de musculação. Neste estudo, os idosos foram submetidos a diferentes testes como o de caminhada de dez metros, levantar-se da posição sentada, levantar-se da posição decúbito ventral, levantar-se da cadeira e locomoverse pela casa, vestir e tirar uma camiseta, e a partir destes resultados os autores calcularam o índice geral de autonomia funcional (IG). Dos avaliados, $100 \%$ obtiveram IG classificado como muito bom, enquanto $71,43 \%$ das avaliadas foram classificadas com IG muito bom e 28,57\% com IG bom, evidenciando que a prática de exercícios de força é capaz de atuar diretamente na autonomia funcional de idosos (SOUZA et al., 2018).

Os trabalhos supracitados corroboram com os resultados obtidos em revisão incluindo 121 estudos clínicos com cerca de 6700 participantes idosos que demonstrou que treinamentos de força (resistência) feitos de 2 a 3 vezes por semana resultam em aumento notável na força muscular, aumento moderado da distância percorrida a pé, melhor desempenho para levantar de uma posição sentada, e uma mobilidade subjetivamente maior. Os autores ainda ressaltam que este tipo de treinamento proporciona o aumento de resistência, aumento da capacidade mitocondrial, enquanto que a frequência cardíaca de repouso diminui (LIU; LATHAM, 2009).

Com relação aos efeitos do exercício resistido, reporta-se a redução do risco de morte por doenças cardiovasculares, a melhora do controle da pressão arterial, no controle do peso corporal, na postura, melhora nos níveis de glicose sanguínea, na função intestinal, nas respostas imunológicas e melhora na qualidade do sono. Além disso, a musculação é especificamente aconselhável na prevenção e auxílio de diversas patologias crônicas degenerativas, tais como: diabetes, hipertensão e possibilita melhora significativa nas funções diárias do idoso, como subir escadas, levantar-se, tomar banho, abrir e fechar portas (RADAELLI et al., 2013). 
Segundo Almeida e Pavan (2010), a musculação é uma ferramenta que auxilia na manutenção e prevenção da saúde psicossocial do idoso, esta atividade é capaz de proporcionar prazer, alegria e descontração, fazendo com que as limitações próprias da idade sejam reduzidas ou até mesmo desapareçam. A prática da musculação possibilita o indivíduo se integrar na sociedade, de modo autônomo e independente, o que contribui para a diminuição da depressão, ansiedade, impaciência, tristeza e solidão, sentimentos próprios desta fase da vida.

Quadro 1. Estudos analisando o treinamento resistido.

\begin{tabular}{|c|c|c|c|c|}
\hline Autor & $\mathrm{N}$ & Objetivos & Metodologia & Conclusões \\
\hline $\begin{array}{l}\text { Cunha et } \\
\text { al. } 2012\end{array}$ & 16 & $\begin{array}{l}\text { Verificaram } 0 \\
\text { efeito de duas } \\
\text { intensidades de } \\
\text { treinamento } \\
\text { resistido sobre a } \\
\text { pressão arterial de } \\
\text { idosas } \\
\text { hipertensas } \\
\text { controladas. }\end{array}$ & 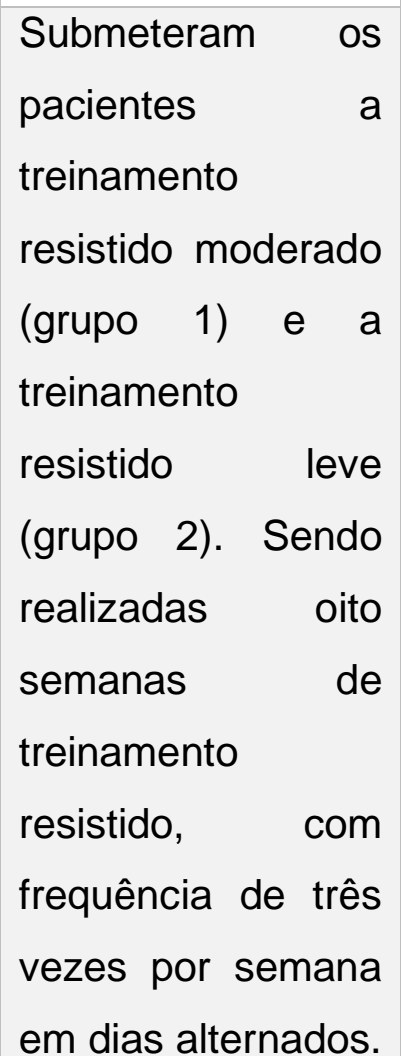 & $\begin{array}{lr}\text { Observaram que tanto o } \\
\text { treinamento resistido } \\
\text { moderador quanto } \\
0 \quad \text { leve } \\
\text { promoveram benefícios } \\
\text { cardiovasculares. }\end{array}$ \\
\hline $\begin{array}{l}\text { Helrigle } \\
\text { et al. } \\
2013\end{array}$ & 86 & $\begin{array}{l}\text { Compararam o } \\
\text { equilíbrio } \\
\text { funcional entre } \\
\text { grupos de idosos } \\
\text { praticantes de } \\
\text { musculação e }\end{array}$ & $\begin{array}{l}\text { Avaliaram o } \\
\text { equilíbrio funcional } \\
\text { a partir do resultado } \\
\text { atingido na Escala } \\
\text { de Equilíbrio de } \\
\text { Berg (EEB) }\end{array}$ & $\begin{array}{l}\text { A prática regular da } \\
\text { musculação, por mais } \\
\text { de seis meses, } \\
\text { promoveu o aumento do } \\
\text { equilíbrio funcional dos } \\
\text { idosos. }\end{array}$ \\
\hline
\end{tabular}




\begin{tabular}{|c|c|c|c|c|}
\hline & & $\begin{array}{l}\text { idosos } \\
\text { sedentários } \\
\text { (ativos e inativos). }\end{array}$ & & \\
\hline $\begin{array}{l}\text { Arruda et } \\
\text { al. } 2014\end{array}$ & 22 & $\begin{array}{l}\text { Avaliaram os } \\
\text { efeitos do treino } \\
\text { de força resistido } \\
\text { isométrico, com e } \\
\text { sem resposta } \\
\text { visual, sobre a } \\
\text { força e função de } \\
\text { idosos. }\end{array}$ & $\begin{array}{l}\text { Realizaram a } \\
\text { aplicação de um } \\
\text { questionário de } \\
\text { qualidade de vida e } \\
\text { do teste de levantar } \\
\text { e sentar, além da } \\
\text { aferição da força } \\
\text { por meio do } \\
\text { esfigmomanômetro } \\
\text { modificado (EM). }\end{array}$ & $\begin{array}{l}\text { Concluíram que o treino } \\
\text { de força resistido } \\
\text { melhorou a qualidade de } \\
\text { vida, a força e a } \\
\text { capacidade funcional de } \\
\text { idosos. }\end{array}$ \\
\hline $\begin{array}{l}\text { Coelho } \\
\text { et al. } \\
2014\end{array}$ & 24 & $\begin{array}{l}\text { Compararam a } \\
\text { força e a } \\
\text { capacidade } \\
\text { funcional entre } \\
\text { idosos praticantes } \\
\text { de musculação e } \\
\text { não praticantes de } \\
\text { exercícios físicos. }\end{array}$ & 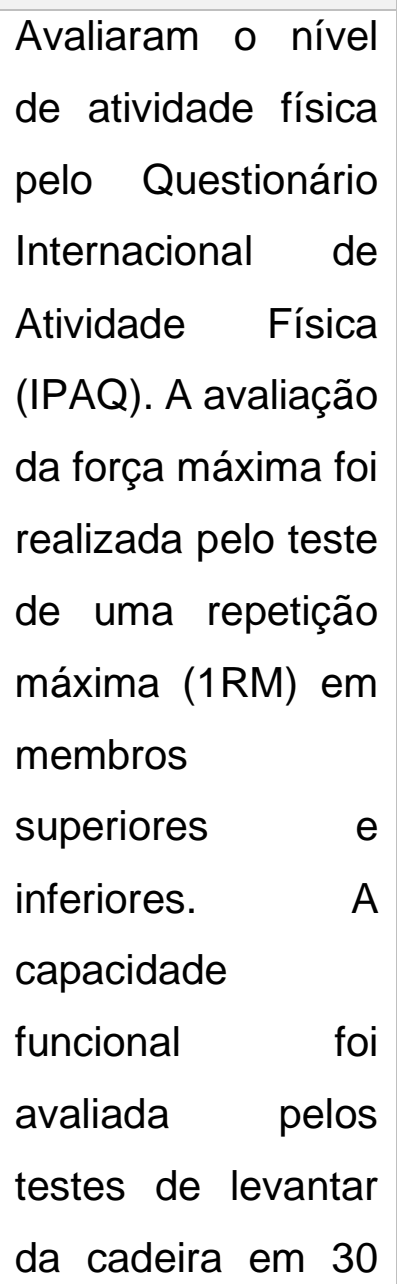 & $\begin{array}{l}\text { Observaram que a } \\
\text { musculação foi eficiente } \\
\text { para o aumento de } \\
\text { força. }\end{array}$ \\
\hline
\end{tabular}




\begin{tabular}{|c|c|c|c|c|}
\hline & & & $\begin{array}{l}\text { segundos } \\
\text { velocidade de } \\
\text { caminhada habitual } \\
\text { e máxima }\end{array}$ & \\
\hline $\begin{array}{l}\text { Silva et } \\
\text { al. } 2016\end{array}$ & 10 & $\begin{array}{l}\text { Verificaram a } \\
\text { influência do } \\
\text { treinamento } \\
\text { resistido sobre a } \\
\text { aptidão } \\
\text { cardiorrespiratória } \\
\text { de idosos }\end{array}$ & $\begin{array}{l}\text { Avaliaram a massa } \\
\text { corporal, } \\
\text { estatura, a força } \\
\text { muscular } \\
\text { isocinética e o } \\
\text { consumo de } \mathrm{O}_{2} \text { em } \\
\text { um teste } \\
\text { cardiorrespiratório } \\
\text { submáximo antes e } \\
\text { após cinco meses } \\
\text { de treinamento de } \\
\text { musculação. }\end{array}$ & $\begin{array}{l}\text { Verificaram que o } \\
\text { treinamento resistido foi } \\
\text { eficaz para } \\
\text { desenvolvimento da } \\
\text { capacidade aeróbia de } \\
\text { idosos. }\end{array}$ \\
\hline $\begin{array}{l}\text { Venâncio } \\
\text { et al. } \\
2016\end{array}$ & 40 & $\begin{array}{l}\text { Analisaram a } \\
\text { influência da } \\
\text { musculação nos } \\
\text { componentes } \\
\text { psicomotores em } \\
\text { idosos }\end{array}$ & $\begin{array}{l}\text { Realizaram estudo } \\
\text { descritivo com } \\
\text { idosos praticantes } \\
\text { de musculação e } \\
\text { sedentários. As } \\
\text { informações foram } \\
\text { coletadas a partir } \\
\text { de testes da ficha } \\
\text { de avaliação } \\
\text { psicomotora de } \\
\text { Rosa Neto que } \\
\text { avalia alterações } \\
\text { de comportamento } \\
\text { mental psicológico } \\
\text { e neurológico. }\end{array}$ & $\begin{array}{l}\text { Observaram que a } \\
\text { prática de musculação } \\
\text { influenciou na melhora e } \\
\text { manutenção dos } \\
\text { componentes } \\
\text { psicomotores } \\
\text { idosos. }\end{array}$ \\
\hline
\end{tabular}




\begin{tabular}{|c|c|c|c|c|}
\hline $\begin{array}{l}\text { Alves et } \\
\text { al. } 2018\end{array}$ & 22 & $\begin{array}{l}\text { Avaliaram de } \\
\text { forma qualitativa } \\
\text { os efeitos dos } \\
\text { exercícios } \\
\text { resistidos } \\
\text { sobre a qualidade } \\
\text { de vida em idosos. }\end{array}$ & $\begin{array}{l}\text { Realizaram a } \\
\text { aplicação de } \\
\text { questionário com } \\
10 \quad \text { perguntas } \\
\text { baseadas } \\
\text { Medical Outcomes } \\
\text { Study no } \\
\text { Categorias de fala: } \\
\text { 1) melhora na } \\
\text { saúde e 2) diálogos } \\
\text { inter-relacionais. }\end{array}$ & $\begin{array}{l}\text { Concluíram, através da } \\
\text { análise qualitativa, que } \\
\text { os exercícios resistidos } \\
\text { melhoram a qualidade } \\
\text { de vida de idosos. }\end{array}$ \\
\hline $\begin{array}{l}\text { Fonseca } \\
\text { et al. } \\
2018\end{array}$ & 9 & $\begin{array}{l}\text { Avaliaram os } \\
\text { efeitos de um } \\
\text { programa de } \\
\text { treinamento de } \\
\text { força } \\
\text { indicadores de } \\
\text { composição } \\
\text { corporal e aptidão } \\
\text { física funcional de } \\
\text { idosos ativos } \\
\text { praticantes } \\
\text { musculação de }\end{array}$ & $\begin{array}{l}\text { Avaliaram a } \\
\text { composição } \\
\text { corporal: Índice de } \\
\text { Massa Corporal, } \\
\text { Perímetro } \\
\text { Abdominal, } \\
\text { Relação } \\
\text { Cintura/Quadril e } \\
\text { Dobras Cutâneas. } \\
\text { Avaliaram também } \\
\text { a aptidão física } \\
\text { funcional: testes de } \\
\text { Levantar da } \\
\text { Cadeira, levantar e } \\
\text { caminhar } 3 \text { metros } \\
\text { e Sentar e Alcançar } \\
\text { (Banco de Wells). }\end{array}$ & $\begin{array}{l}\text { Observaram que } \\
\text { treinamento periodizado } \\
\text { de força é eficiente na } \\
\text { melhoria dos índices de } \\
\text { aptidão física funcional e } \\
\text { composição corporal de } \\
\text { idosos. }\end{array}$ \\
\hline
\end{tabular}




\section{CONSIDERAÇÕES FINAIS}

O treinamento resistido (musculação) deve ser incentivado dentre a população idosa e sempre acompanhado por um profissional habilitado na elaboração dos programas de treinamento, objetivando melhorar a qualidade de vida e as valências físicas do indivíduo durante o envelhecimento.

A prática de musculação, com periodicidade de no mínimo duas vezes por semana traz bons resultados para a vida tanto de homens quanto de mulheres idosas. Os resultados incluem benefícios cardiovasculares, desenvolvimento da capacidade aeróbia, melhora na composição corporal, aumento do equilíbrio funcional, aumento da força e capacidade funcional, melhora nos índices de aptidão física e melhora dos componentes psicomotores.

Conforme demonstrado, a musculação proporciona aumento na força muscular e na resistência do idoso, melhorando seu equilíbrio e a sua capacidade cognitiva. Estas mudanças fisiológicas são essenciais para que o indivíduo consiga realizar tarefas do seu dia a dia de maneira mais autônoma e independente, recuperando assim sua autoconfiança e autoestima.

\section{REFERÊNCIAS}

AGUIAR, P. DE P. L.; LOPES, C. R.; VIANA, H. B., GERMANO, M. D. Avaliação da influência do treinamento resistido de força em idosos. Revista Kairós Gerontologia. v. 17, n. 3, p. 201-217, 2014.

ALMEIDA, M.A.B. DE; PAVAN, B. Os benefícios da musculação para a vida social e para o aumento da auto-estima na terceira idade. Revista Brasileira de Qualidade de Vida. v. 02, n. 02, p. 09-17, 2010.

ARAÚJO, L. C. C. de. A prática da musculação na melhor idade: Uma revisão bibliográfica. 2017.17 p. Curso de Educação Física (Trabalho de Conclusão de Curso) da Universidade Estadual da Paraíba. Campina Grande, Paraíba. 
CANNON, J.; KAY, D.; TARPENNING, K.M.; MARINO, F.E. Comparative effects of resistance training on peak isometric torque, muscle hypertrophy, voluntary activation and surface EMG between young and elderly women. Clinical Physiology and Functional Imaging. 27(2), 91-100, 2007.

CORAZZA, M. A. Terceira Idade \& Atividade Física. 1. ed., São Paulo: Phorte, 2001.

DIAS, R. M. R.; GURJÃO, A. L. D.; MARUCCI, M. F. N. Benefícios do treinamento com pesos para aptidão física de idosos. Acta fisiátrica. v. 13, n. 2, p. 90-95, 2016.

JAREK, C.; OLIVEIRA, M. H.; NANTES, W. R.; ULBRICHT, L.; MASCARENHAS, L. P. G. Comparação antropométrica, força muscular e equilíbrio entre idosos praticantes e não praticantes de musculação. RBCEH. v. 7, n. 2, p. 173-180, 2010.

KIRKWOOD, R.N. et al. Análise biomecânica das articulações do quadril e joelho durante a marcha em participantes idosos. Revista Brasileira de Fisioterapia, v. 15, n. 5, p. 267-271, 2007.

LIU, C.J.; LATHAM, N.K. Progressive resistance strength training for improving physical function in older adults. Cochrane Database of Systematic Reviews. v. 8, n. 3, 2009.

PEDRO, E. M.; AMORIM, D. B. Análise comparativa da massa e forca muscular e do equilíbrio entre indivíduos idosos praticantes e não praticantes de musculação. Conexões. v. 6, n. esp., p. 174-183, 2008.

RADAELLI, R.; BOTTON, C.E.; WILHELM, E.N.; BOTTARO, M.; LACERDA, F.; GAYA, A.; MORAES, K.; PERUZZOLO, A.; BROWN, L.E.; PINTO, R.S. Low-and highvolume strength training induces similar neuromuscular improvements in muscle quality in elderly women. Experimental Gerontology. v. 48, n. 8, p. 710-716, 2013.

SMOLAREK, A. DE C.; FERREIRA, L. H. B.; MASCARENHAS, L. P. G.; MCANULTY, S. R.; VARELA, K. V.; DANGUI, M. C.; BARROS, M. P. DE; UTTER, A. C.; SOUZA- 
JUNIOR, T. P.The effects of strength training on cognitive performance in elderly women. Clinical Interventions in Aging. v. 11, p. 749-754, 2016.

SOUZA, L. K. DE; COELHO, B. DOS S.; FREIRE, B.; DELEVATTI, R. S.; RONCADA, C.; TIGGEMANN, C. L.; DIAS, C. P. Comparação dos níveis de força e equilíbrio entre idosos praticantes de musculação e de hidroginástica. Revista Brasileira de Atividade Física e Saúde. v. 19, n. 5, p. 647-648, 2014.

SOUZA, A. DE O. DE; PEDROSO, B. B.; SIQUEIRA, O. D.; GARLIPP, D. C. Avaliação da autonomia funcional de idosos praticantes de Musculação. Revista de Iniciação Científica da Ulbra. n. 16, p. 52-59, 2018

SOUZA, Lucas et al. Comparação dos níveis de força e equilíbrio entre idosos praticantes de musculação e de hidroginástica. Revista Brasileira de Atividade Física \& Saúde, v. 19, n. 5, p. 647-647, 2014.

WHO - World Health Organization. Ageing and life-course. Disponível em: $<$ https://www.who.int/ageing/healthy-ageing/en/> Acesso em: 01/04/2019.

Enviado: Agosto, 2020.

Aprovado: Setembro, 2020. 